\title{
Sensing Performance of Advanced LTE Cognitive Femtocells
}

\author{
Mohamed Shalaby \\ National Institute for Standards \\ Egypt
}

\author{
Mona Shokair \\ Faculty of Electronic Engineering \\ Menoufia University \\ Egypt
}

\author{
Yaser S. E. Abdo \\ National Institute for Standards \\ Egypt
}

\begin{abstract}
The deployment of femtocells in a LTE advanced system can extend the system coverage to include indoors. Unfortunately, an electromagnetic interference may occur among the femto users and the macro users in the case of co-channel deployment. A cognitive radio can be used to mitigate the interference among the femtocells and the macrocells. It is applied by allowing the femto users to be handled as secondary users of any other existing network. In this paper, the performance of the LTE advanced Femtocell is studied by using different detectors such as energy detector, cyclostationary detector, and matched filter detector, which is not clarified until now. Moreover, the analysis of these detectors is made. Comparisons among these detectors are carried out. Different wireless channel models, Additive White Gaussian Noise (AWGN) and fading channels, are implemented to verify the operation of the proposed LTE advanced Femtocell. Simulation results show that there is a tradeoff between a false alarm probability and the signal to noise ratio value of any detector to have a certain performance. Moreover, the performance of the cyclostationary detector and the matched filter detector is better than the energy detector especially at low signal to noise ratio values. Unfortunately, the cyclostationary detector performance is not satisfying when the fading channels are employed.
\end{abstract}

\section{Keywords}

LTE Advanced, Femtocells, Cognitive Radio, Spectrum Sensing, Wireless Channel Models

\section{INTRODUCTION}

A LTE advanced is a new broad band technology which has a data rate of $1 \mathrm{Gbps}$ [1]. This data rate can be achieved through different techniques, which were discussed before in Ref. [2]. To extend the system coverage to include indoors, femtocells can be used. They are small base stations that emit low power in order to cover small range. These small base stations are preferable than other techniques to improve the system coverage [3]. Since the femtocells become an important technology, it is supported by standards of 3GPP [4, and 5].

Femtocells usually share the spectrum with the macrocells in such a way that, the available spectrum is accessed by the macro users and the femto users. Therefore, an electromagnetic interference may occur among them. This spectrum allocation is called a co-channel deployment or a universal frequency reuse. This mutual interference can degrade the user's capacity. One of the methods, which are used to mitigate this interference, is applying the cognitive radio. The available spectrum is allocated to the macrocells and the femtocells are allowed to operate as secondary users at the spectrum holes of any other existing fixed policy wireless network. The femto users have to sense the wide band spectrum in order to allocate the empty slots within this spectrum. Spectrum sensing can be achieved by a lot of techniques. One of these techniques is the transmitter detection such as the energy detector, the cyclostationary detector, and the matched filter detector [6, 7, and 8].

In this paper, the performance of the LTE femtocells is studied by using three different detectors which are not clarified in the published literature until now. The analysis of these detectors is made. Moreover, the performance is estimated after employing an AWGN channel and the fading channels. Also, comparisons among different spectrum sensing techniques are carried out. The spectrum sensing process of the cognitive radio is explained in Section 2 . Subsequently, different models, which are used to represent the wireless channel behavior, are stated in Section 3. Finally, the simulation results are discussed in Section 4.

\section{SPECTRUM SENSING TECHNIQUES}

A spectrum sensing is the most important stage in the cognitive radio system. If it is not carried out properly, the cognitive system will not be successful [6, 7, and 8]. Assuming $x(t)$ is the primary user signal and $y(t)$ is the received signal at the secondary user receiver. The received signal at the secondary user can be considered as;

$$
\begin{array}{r}
y(t)=n(t) \quad H_{o} \\
y(t)=h x(t)+n(t) \quad H_{1}
\end{array}
$$

where $H_{o}$ denotes that the primary user signal is absent, and $H_{l}$ gives an indication of the signal presence. There are three parameters, which are used to evaluate the performance of any detector, and they are;

- Probability of detection: the capability of the detector to detect a user, who is really active in a certain frequency band. This has a great role to mitigate the interference between the primary user and the secondary one.

$$
p_{d}=p_{r}\left(y \succ \lambda \mid H_{1}\right)
$$

- Probability of false alarm: the detector indicates that the primary user is present but, he is really absent. This leads the spectrum to be wasted.

$$
p_{f a}=p_{r}\left(y \succ \lambda \mid H_{o}\right)
$$


- Probability of missed detection: the primary user is active but, the detector indicates that he is absent. This results in a severe interference between the primary user and the secondary one.

$$
p_{m d}=p_{r}\left(y \prec \lambda \mid H_{1}\right)
$$

where $\lambda$ is a threshold value. Primary users can be detected using different methods, which will be explained through the following subsections.

\subsection{Energy Detector}

Estimation of the received signal energy is the basic principle of the energy detector. Figure 1 presents the energy detector in the time domain as in Ref. [9]. The filter selects the desired signals and neglects any signal or noise, which is out of the band. The filtered signal samples are squared to obtain the power of the signal. When the integrator is added, the signal energy can be calculated. The obtained energy is compared to a threshold value $(\lambda)$ to decide, if a primary user is present or not.

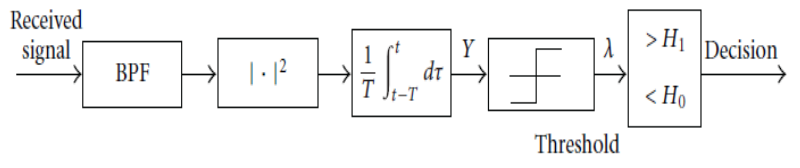

Fig 1: The block diagram of the energy detector [9]

The received signal at the cognitive user can be expressed as;

$$
y(t)=\frac{1}{T} \int_{t-T}^{t}|x(\tau)|^{2} d \tau
$$

The detection probability of the energy detector is given by;

$$
p_{d}=p_{r}\left(y \succ \lambda \mid H_{1}\right)=Q_{u}(\sqrt{2 \gamma}, \sqrt{\lambda})
$$

where $\lambda$ is the threshold value, $\gamma$ is the signal to noise ratio, $2 u$ is the number of samples, and $Q_{M}$ is the Marcum $Q$-function of order $M$. For any parameters such as $\alpha$ and $\beta$, it can be evaluated by;

$$
Q_{M}(\alpha, \beta)=\int_{\beta}^{\infty} \frac{t^{M}}{\alpha^{M-1}} e^{\frac{-\left(t^{2}+\alpha^{2}\right)}{2}} I_{M-1}(\alpha t) d t
$$

where $I_{M-1}($.$) is the modified bessel function of (M-1)^{\text {th }}$ order. The false alarm probability can be obtained by;

$$
p_{f a}=p_{r}\left(y \succ \lambda \mid H_{o}\right)=\frac{\Gamma\left(u, \frac{\lambda}{2}\right)}{\Gamma(u)}
$$

where $\Gamma\left(u, \frac{\lambda}{2}\right)$ is the incomplete gamma function, that can be expressed as;

$$
\Gamma(m, n)=\int_{n}^{\infty} t^{m-1} e^{-t} d t
$$

and $\Gamma(u)$ is the gamma function, which can be got by;

$$
\Gamma(u)=\int_{0}^{\infty} t^{u-1} e^{-t} d t
$$

The missed detection probability of the energy detector can be given by;

$$
p_{m d}=1-p_{d}=1-Q_{u}(\sqrt{2 \gamma}, \sqrt{\lambda})
$$

The energy detector has an easy design, need no priori information about the primary users' signals, and has a low computational complexity. On the other hand, the energy detector has several drawbacks such as; it has a high false alarm probability due to the noise uncertainty, it cannot distinguish among the primary users' signals, and it has a poor performance, when the fading channels and shot noise are implemented [6,7, and 8].

\subsection{Matched Filter Detector}

When prior information about the primary user signal is present, the matched filter performance becomes very good. It has the ability to increase the signal to noise ratio, especially, at the sampling instant. Supposing that, the primary user signal is $X(n)$ and $Y(n)$ is a known replica at the secondary receiver, then the correlation function exists. The correlation function $T(x)$, as assumed in Ref. [6], is;

$$
T(x)=\sum_{n=0}^{N-1} X(n) Y(n)
$$

The false alarm probability, as discussed in Ref. [7], becomes;

$$
p_{f a}=p_{r}\left(T(x) \succ \beta \mid H_{o}\right)=Q\left(\beta / \sqrt{\frac{\sigma^{2}}{N}}\right)
$$

where $\beta$ is the threshold value, $N$ is the number of samples needed to achieve an optimum detection, $\sigma^{2}$ is the noise variance, and $Q(x)$ is the standard $Q$ function, which can be estimated by;

$$
Q(x)=\int_{x}^{\infty} \frac{1}{\sqrt{2 \pi}} e^{\frac{-t^{2}}{2}} d t
$$

Since the matched filter maximizes the signal to noise ratio at the sampling instant, the maximum of the correlation function is considered to be at the sampling instant. Then, it is compared to the threshold value, which can be determined from the false alarm probability (Eq.13), to decide if the primary user is present or not.

The matched filter detector has a very good performance, especially, at low signal to noise ratio values, and very immune to noise. On the other hand, prior information about the primary user signal has to be available $[6,7$, and 8$]$.

\subsection{Cyclostationary Detector}

The cyclostationary detector can distinguish signals from noise, on the basis that the transmitted signals are periodic with a spectral correlation, but the noise is a random signal with no correlation. The signal is cyclostationary, if the mean and autocorrelation function are periodic;

$$
E\left\{x\left(t+T_{o}\right)\right\}=E\{x(t)\}
$$

where $E$ is the expectation (mean), $T_{0}$ is the signal period.

$$
R_{x}\left(t+T_{0}, \tau\right)=R_{x}(t, \tau)
$$


where $R_{x}$ is the autocorrelation function and can be estimated by; $R_{x}=E\{x(t+\tau) x(t)\}$

The signal becomes cyclostationary, therefore, the cyclic autocorrelation function exists, which can be expressed as;

$$
R_{x}^{\alpha}(\tau)=\frac{1}{T_{o}} \int_{\frac{-T_{O}}{2}}^{\frac{T_{O}}{2}} R_{x}(t, \tau) e^{-j 2 \pi \alpha t} d t
$$

where $\alpha$ is the cycle frequency, it may be the carrier frequency or its harmonics. The spectral correlation function is;

$$
S(f, \alpha)=f f t\left(R_{x}^{\alpha}(\tau)\right)=\int_{-\infty}^{\infty} R_{x}^{\alpha}(\tau) e^{-j 2 \pi \tau \tau} d \tau
$$

Figure 2 shows the block diagram of the cyclostationary detector, as discussed before in Ref. [8]. FFT can be used to obtain the spectral components of the digital received signal. The average of the spectral correlation (Eq.18) is compared to a threshold value to decide if the primary user is present or absent. The spectral correlation function describes the relation among the signal samples separated by the cycle frequency [10].

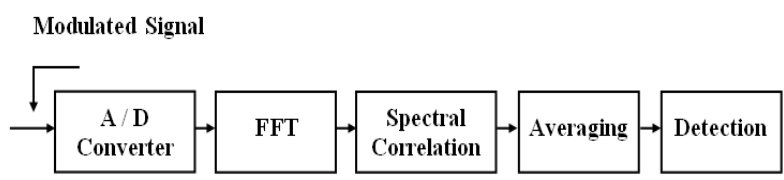

Fig 2: The block diagram of the cyclostationary detector [8]

The spectral correlation function is obtained and the summation of its components is normalized by the frame length $K$, and the maximum is compared to the threshold value $d$, which is a function of the false alarm probability.

$$
S_{x}^{\alpha}=\frac{1}{K W} \sum_{i=1}^{K} S_{x i}^{\alpha}
$$

where $W=\|$ window $w \|^{2}$ is the window length.

$$
C=\max \left(S_{x}^{\alpha}\right)
$$

The maximum of the spectral correlation function (C) is compared to the threshold value. The false alarm probability can be expressed as [11, and 12];

$$
p_{f a}=(1-d)^{N-1}
$$

where $N$ is the number of samples needed to achieve an optimum detection. The cyclostationary detector has a good performance at low signal to noise ratio values, can differentiate among the primary users' signals. On the contrary, it is complex and needs a long observation time [6, 7 , and 8].

\section{WIRELESS CHANNEL MODELS}

The wireless channel behavior is not stationary but it becomes different from time to time. Wireless channel models can provide an acceptable approximation of the channel behavior. There are a lot of models that can describe the wireless channel behavior; such as the AWGN, Rayleigh fading, and
Rician fading models, which are the most famous models of fading.

\subsection{Additive White Gaussian Noise}

It is the simplest model which can describe the wireless channel behavior [13]. A random signal is added to the transmitted signal to represent the channel effect. Suppose that $x(t)$ is the transmitted signal, the received signal $s(t)$ can be expressed as;

$$
s(t)=x(t)+n(t)
$$

where $n(t)$ is the noise signal.

\subsection{Fading Channels}

A multipath propagation and a Doppler spread are the main causes of fading [13, and 14]. The signal arrives to the receiver from different paths having different delays and different path gains. Moreover, these paths have different frequency shifts. These paths of propagation can weak the signal arrived at the receiver. The received signal is the algebraic summation of the different paths of propagation.

\subsubsection{Rayleigh Fading Model}

The Rayleigh fading can model the wireless channel, when there is no direct line of sight between the transmitter and the receiver. This fading model is usually used to describe the mobile channel behavior. The probability density function $f(r)$ of Rayleigh fading is given by;

$$
f(r)=\frac{r}{\sigma^{2}} \exp \left(-\frac{r^{2}}{2 \sigma^{2}}\right) \quad r \geq 0
$$

where $r$ is the envelope and $\sigma^{2}$ is the variance.

\subsubsection{Rician Fading Model (Nakagami-n model)}

The existence of a strong dominant path between the transmitter and the receiver is the major difference between Rician and Rayleigh fading models. This path is called the LOS (Line of Sight) path. The envelope, $r$, of a Rician fading has a probability density function, $f(r)$, which can be evaluated by;

$$
f(r)=\frac{r}{\sigma^{2}} \exp \left(-\frac{r^{2}+k_{d}^{2}}{2 \sigma^{2}}\right) I_{o}\left(\frac{r k_{d}}{\sigma^{2}}\right) \quad r \geq 0
$$

where $I_{o}()$ is the $0^{\text {th }}$ order modified Bessel function of the first kind, $k_{d}$ is the strength of the LOS component. A Rician fading can be reduced to a corresponding Rayleigh one, when $k_{d}=0$.

\section{SIMULATION RESULTS}

The energy detector, the cyclostationary detector, and the matched filter detector are simulated employing AWGN channel and the fading channels. The performance of the simulated detectors is validated as the probability of detection versus the false alarm probability. The probability of detection is estimated by allowing a primary user to send a random data 1000 times, and counting the times that the cognitive user can detect this signal. The probability of detection can be calculated as the detection times divided by the sending times. Table 1 contains the simulation parameters.

The performance of the three detectors implementing AWGN, Rayleigh fading, and Rician fading is estimated. Figure 3(a) presents the detection probability versus the false alarm probability of the cyclostationary detector at the signal to 
noise ratio value of $-6 \mathrm{~dB}$. Figure $3(\mathrm{~b})$ shows the performance of the energy detector at the signal to noise ratio value of $0 \mathrm{~dB}$ while Figure 3(c) displays the matched filter performance at the signal to noise ratio value of $-12 \mathrm{~dB}$. From Figure 3 , it is obvious that the performance of any detector is better employing an AWGN channel than fading channels. When the false alarm probability increases, the probability of detection becomes better. The matched filter detector is very tolerable to noise and has a satisfied performance when the fading channels are utilized. The fading channels can degrade the cyclostationary detector performance. However, it has a good performance using an AWGN channel.

Table 1. The simulation parameters

\begin{tabular}{|c|c|}
\hline \multicolumn{2}{|c|}{ Simulation Parameters } \\
\hline Sampling Frequency & $1 \mathrm{GHz}$ \\
\hline Carrier Frequency & $95 \mathrm{MHz}$ \\
\hline Sample Points & 10 \\
\hline Input Data & Random \\
\hline Modulation Type & QAM-16 \\
\hline Cycle Frequency & $95 \mathrm{MHz}$ \\
\hline Doppler Frequency & $100 \mathrm{~Hz}$ \\
\hline Rician Fading Parameter & 10 \\
\hline
\end{tabular}

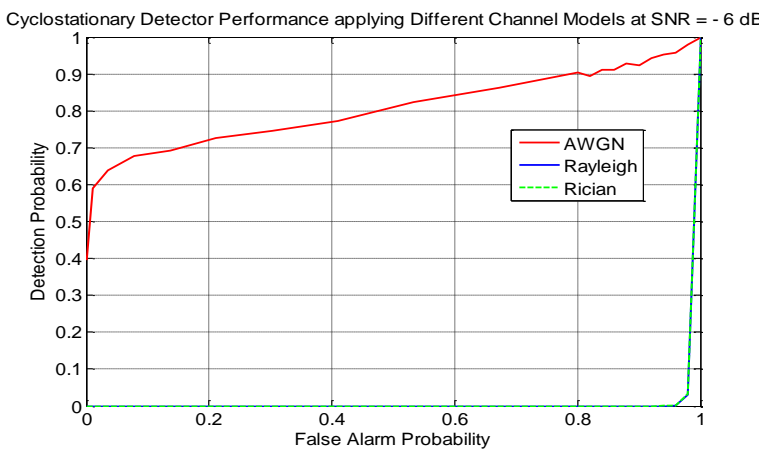

(a)

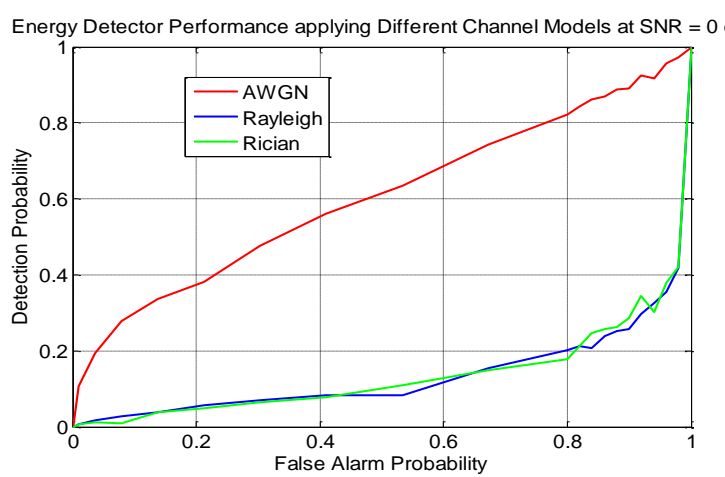

(b)

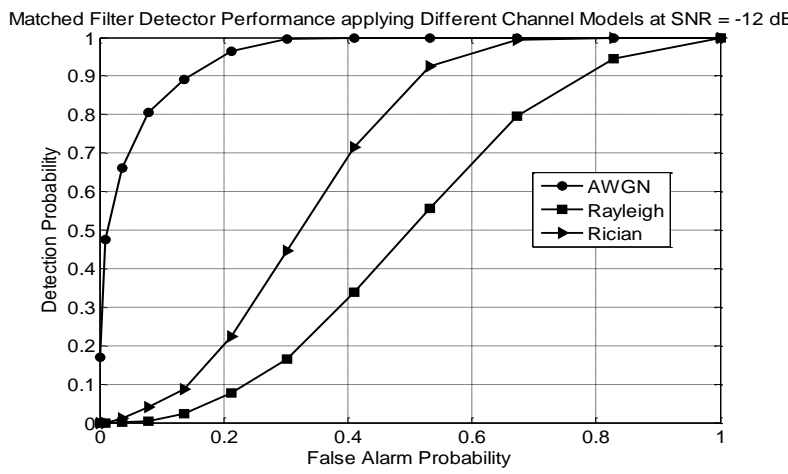

(c)

Fig 3: The performance of the three detectors employing an AWGN and the fading channels

The performance of the three detectors using the fading channels is illustrated in Figure 4. It is clear that, increasing the signal to noise ratio value (SNR) improves the detection probability over the fading channels. Moreover, the performance of the cyclostationary detector is very poor after applying the fading channels. The matched filter detector has a good performance, when the fading channels are employed.

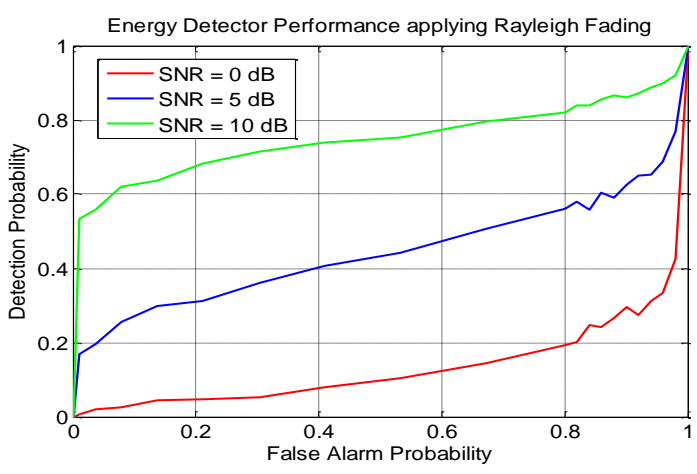

(a)

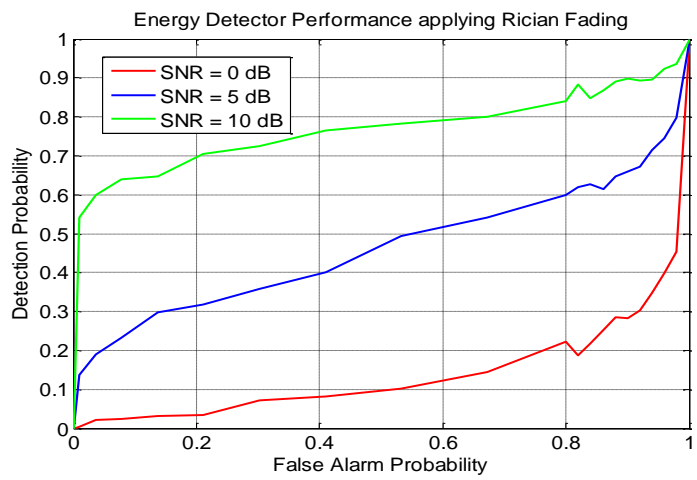

(b) 


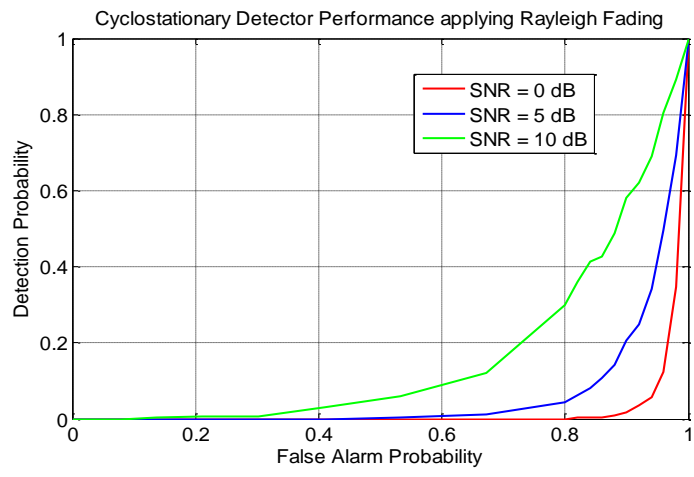

(c)

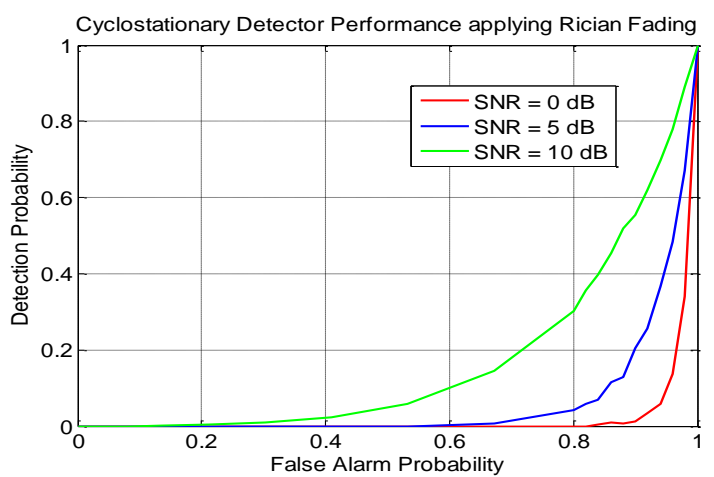

(d)

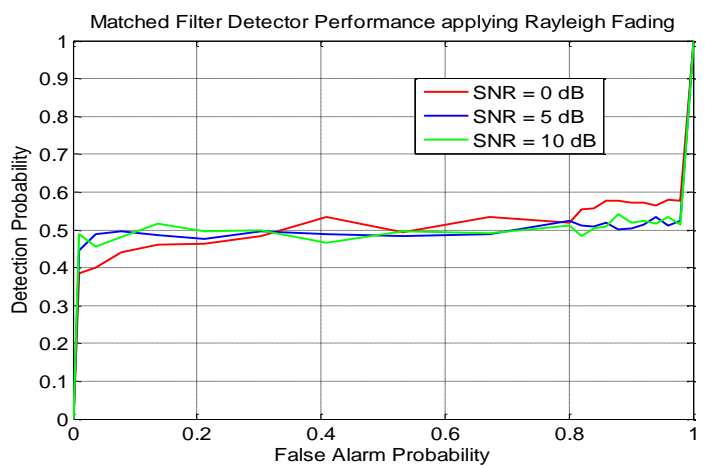

(e)

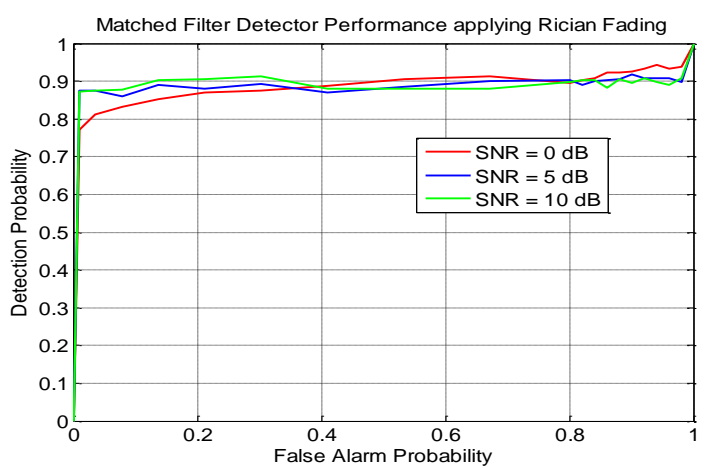

(f)

Fig 4: The performance of the three detectors when the received signals are faded

The effect of the false alarm probability on the performance of the three detectors applying AWGN is explained in more details. Figure 5 displays the performance of the three detectors at different values of the false alarm probability. The values of false alarm probability were selected after running the program several times. It is clear that, the probability of detection of any detector increases, when the false alarm probability increases, and this agrees with the discussion of figure 3. It can be concluded that, for any detector, there is a tradeoff between the false alarm probability value and the signal to noise ratio to achieve a certain performance. Let the detector detect the weak signals, it becomes more prone to the false alarms.

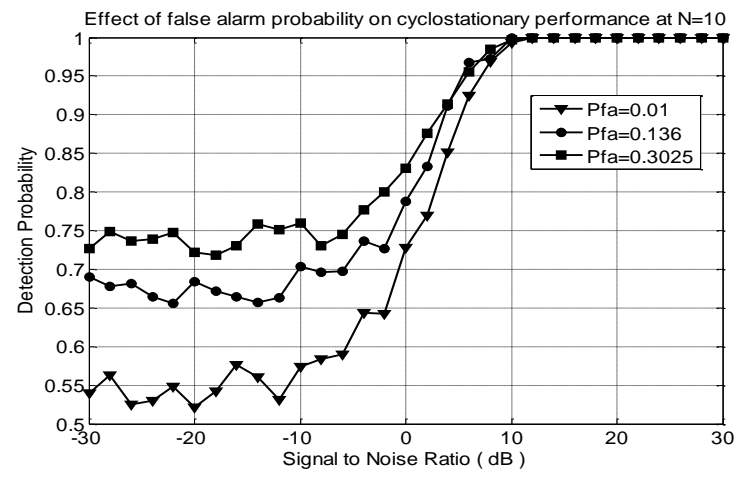

(a)

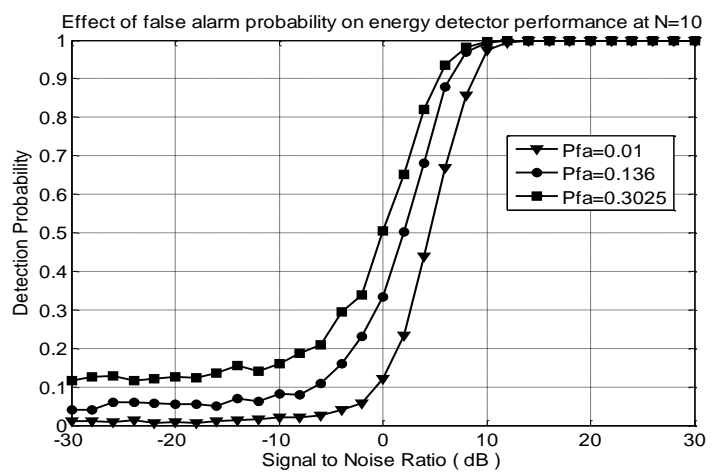

(b)

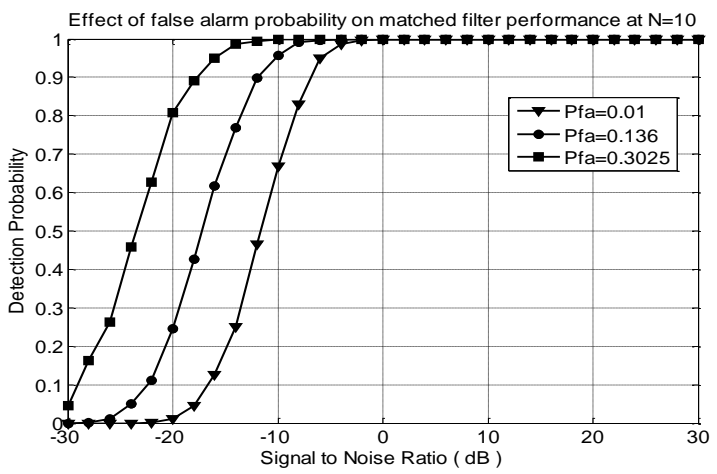

(c)

Fig 5: The false alarm probability effect on the three detectors' performance

The effect of the number of samples on the performance of the three detectors is investigated. Figure 6 presents the performance of the three detectors at different number of samples. It is obvious that, the performance of any detector becomes better, when the number of samples increases. It is 
noticed that the matched filter detector performance is improved sharply for a small increase in the number of samples due to the high correlation. Moreover, the cyclostationary detector performance increases with a small increase in the number of samples especially, at low SNR values, but this performance improvement is less than the matched filter. The energy detector performance increases also, when the number of samples increases, but the improvement is less than other detector types. It can be concluded that, there is a tradeoff between the number of samples used in the detection process and the SNR ratio value for the same performance. Detecting signals at low SNR values requires more samples, which results in more sensing time. Detection can be simply designed for a small number of samples, and hence the sensing time of the signals arrived with higher SNR values is reduced.

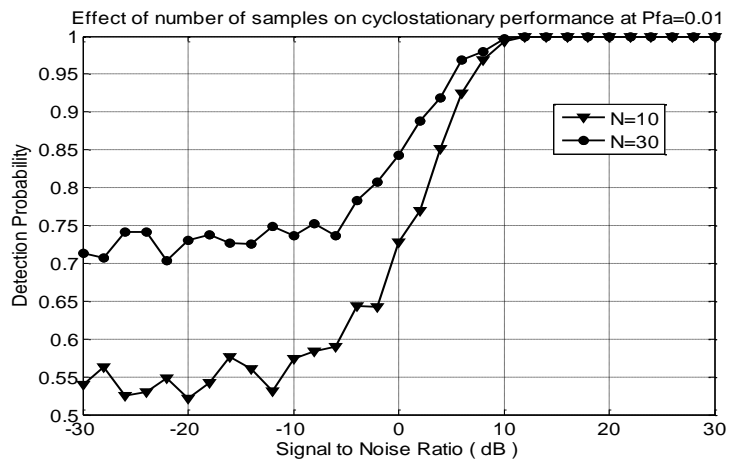

(a)

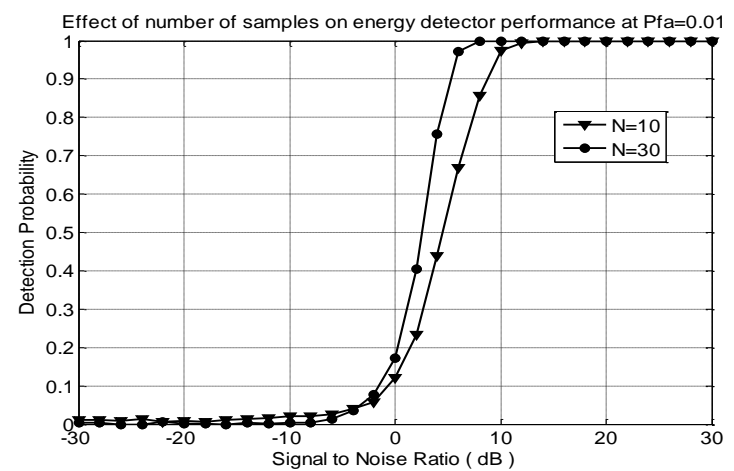

(b)

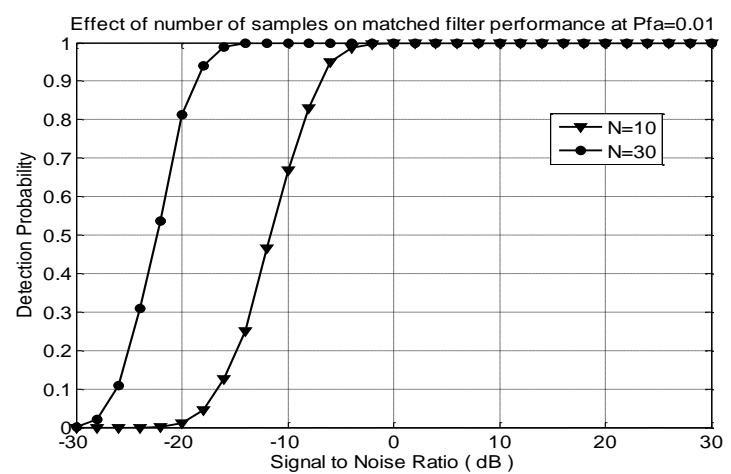

(c)

Fig 6: The three detectors performance variation with the number of samples
In this section, a comparison among the three detectors performance is carried out. From Figure 7, which shows the performance comparison, it can be concluded that the performance of the cyclostationary detector and the matched filter detector is better than the energy detector performance at any conditions. Both of them perform well at low SNR values. Moreover, the matched filter detector performance is improved sharply with the increase in SNR values due to the high correlation.

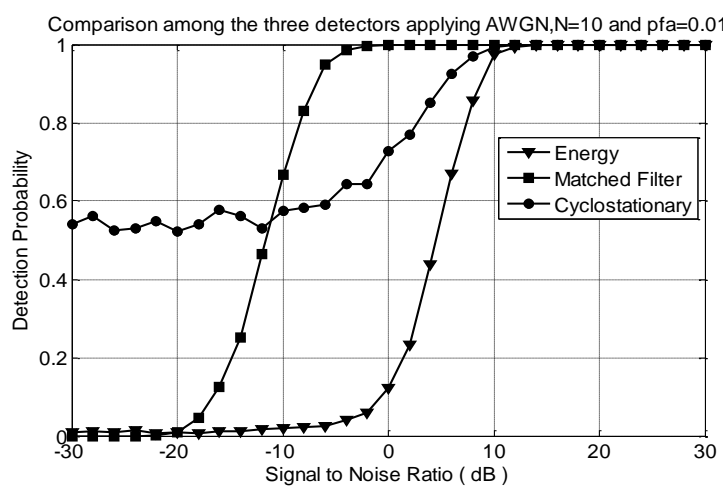

(a)

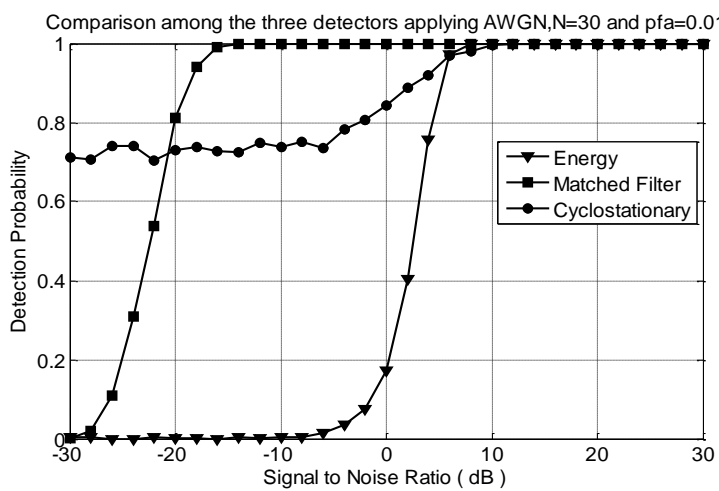

(b)

Fig 7: The comparison among the three detectors after implementing an AWGN

\section{CONCLUSIONS}

In this paper, a comparison among different spectrum sensing methods, which the femto user can use to achieve the cognition process, was held. It can be concluded that, the matched filter has a very good performance at AWGN and an acceptable performance when the received signals are faded. The energy detector has a poor performance and needs a large number of samples to achieve a good performance. Although, the cyclostationary detector performs well at noisy channels, it has a very poor performance when the received signals are faded. If a priori information about the primary user signals is available, the matched filter is the best detector to be used. Tolerating a noise at any detector can increase its false alarms. Moreover, any detector can detect weak signals easily by receiving a large number of samples.

\section{ACKNOWLEDGMENTS}

I am grateful to my supervisors who helped me a lot to complete this work. This work appears in a beautiful form due to their assistance, encouragement, and patience. 


\section{REFERENCES}

[1] D. M. Sacristan, J. F.Monserrat, J. C. Penuelas, D. Calabuig, S. Garrigas, and N. Cardona, "on The Way towards Fourth-Generation Mobile: 3GPP LTE and LTEAdvanced, " EURASIP Journal on Wireless Communications and Networking, Vol. 2009, No. 354089, pp. 1-10, June 2009.

[2] S. Parkvall, E. Dahlman, A. Furuskar, Y. Jading, M. Olsson, S. Wanstedt, and K. Zangi, "LTE-AdvancdEvolving LTE towards IMT-Advanced, " IEEE Vehicular Technology Conference, pp. 1-5, September 2008.

[3] V. Chandrasekhar, and J. G. Andrews, "Femtocell Networks: A Survey, " IEEE Communications Magazine, pp. 59-67, September 2008.

[4] D. N. Knisely, T. Yoshizawa, and F. Favichia, "Standardization of Femtocells in 3GPP, " IEEE Communications Magazine, pp. 68-75, September 2009.

[5] D. N. Knisely, and F. Favichia, "Standardization of Femtocells in 3GPP2, " IEEE Communications Magazine, pp. 76-82, September 2009.

[6] C. Xiao, and A. Kataria, "Cognitive Radios-Spectrum Sensing Issues, "Thesis, Faculty of the Graduate School at the University of Missouri-Columbia, pp. 1-45, December 2007.

[7] H. Hu, "Cyclostationary Approach to Signal Detection and Classification in Cognitive Radio Systems, "Beijing University of Posts and Telecommunication P. R. China, pp. 51-76, 2009.
[8] V. Sonmezer, M. Tummala, and D. Jenn, " Cooperative Wideband Spectrum Sensing and Localization using Radio Frequency Sensor Networks, " thesis, Naval Post Graduate School, Monterey, California, pp. 1-91, September 2009.

[9] H. Rasheed, and N. Rajatheva, "Spectrum Sensing for Cognitive Vehicular Networks over Composite Fading, " International journal of vehicular technology, Vol. 2011, No. 630467, pp. 1-9, January 2011.

[10] M. B. Dave, and S. k. Patra, "Spectrum Sensing in Cognitive Radio: Use of Cyclo-Stationary Detector, " thesis, Electronics and communication Engineering department, National Institute of Technology, India, pp. 1-59, May 2012.

[11] S. Enserink, and D. cochran, "Cyclostationary Feature Detector, "IEEE Conference Record of the TwentyEighth Asilomar on Signals, Systems, and Computers, pp. 806-810, October 1994.

[12] S. Enserink, and D. cochran, "on Detection of Cyclostationary Signals, "IEEE Acoustics, Speech, and Signal Processing Conference, pp. 2004-2007, May 1995.

[13] A. S. Babu, and K. V. S. Rao, "Evaluation of BER for AWGN, Rayleigh, and Rician Fading Channels under Various Modulation Schemes, " International Journal of Computer Applications, Vol. 26, No. 9, pp. 23-28, July 2011.

[14] G. S. Prabhu, and P. M. Shankar, "Simulation of Flat Fading using MATLAB for Classroom Instruction, " IEEE Transactions on Education, Vol. 45, No. 1, pp. 1925, Febuary 2002. 